\title{
NOTA/NOTE
}

\section{La ictiofauna del área marina de la Vía Parque Isla Salamanca, Magdalena, Colombia}

\section{Ichthyofauna of the marine area of Vía Parque Isla Salamanca, Magdalena, Colombia}

\author{
Diana C. Sánchez-Rodríguez ${ }^{{ }^{*}}$, Arturo Acero P. ${ }^{2}$, Efraín Viloria-Maestre ${ }^{3}$, Francisco A. Villa-Navarro ${ }^{4}$ y Patricia Saldaña \\ $\begin{array}{lllll}\text { (D) } 0000-0002-7233-9674 & \text { (D) } 0000-0002-6637-9901 & \text { (D) } 0000-0003-2905-7310 & \text { (D) } 0000-0001-5913-188 X & \text { (D) } 0000-0002-7349-4908\end{array}$ \\ 1. Programa de Biología, Facultad de Ciencias, Universidad del Tolima, Ibagué, Colombia. biologist0104@gmail.com \\ 2. Instituto de Estudios en Ciencias del Mar (Cecimar), Universidad Nacional de Colombia sede Caribe, playa Salguero, Santa Marta, Colombia. \\ aacerop@unal.edu.co \\ 3. Instituto de Investigaciones Marinas y Costeras “José Benito Vives de Andréis” (Invemar), playa Salguero, Santa Marta, Colombia. \\ efrain.viloria@invemar.org.co \\ 4. Facultad de Ciencias, Universidad del Tolima, Ibagué, Colombia.favilla@ut.edu.co \\ 5. Unidad Administrativa de Parques Nacionales Naturales de Colombia, Via Parque Isla de Salamanca, Magdalena, Colombia. \\ patricia.saldana@parquesnacionales.gov.co \\ * Autora de correspondencia.
}

\section{RESUMEN}

$\mathrm{E}$ ntre 2006 y 2007 se realizaron muestreos mensuales de las capturas de peces obtenidas con chinchorro de arrastre tradicional, donde se registró una porción importante de la ictiofauna caribeña de la plataforma continental: 10114 individuos pertenecientes a 16 familias y 31 especies. Las familias Carangidae y Sciaenidae se mencionan entre las de mayor representación del Caribe, mientras que la especie más abundante fue Oligoplites saurus. Del listado de especies, siete se encuentran incluidas en el Libro Rojo de Peces Marinos de Colombia; cinco entre las categorías de En Peligro Crítico (CR) y Vulnerable (VU), y dos en la categoría Casi Amenazada (NT). Estos resultados son un soporte técnico y científico para la toma de decisiones, cumpliendo así con la misión y gestión de conservación para la cual ha sido creada esta área protegida.

PALABRAS CLAVE: Caribe colombiano, áreas marinas protegidas, fauna íctica, pesca artesanal

\section{ABSTRACT}

$\mathrm{M}$ onthly samplings of fish catches obtained by traditional beach seine were carried out between 2006 and 2007, recording an important portion of the Caribbean ichthyofauna of the continental shelf: 10114 individuals belonging to 16 families and 31 species. The families Carangidae and Sciaenidae are mentioned among the most widely represented in the Caribbean, while the most abundant species was Oligoplites saurus. From the list of species, seven are included in the Red Book of Marine Fishes of Colombia; five between the categories Critically Endangered (CR) and Vulnerable (VU), and two in the category Near Threatened (NT). These results are a technical and scientific input for decision making, to fulfill the conservation and management mission which was the aim when this protected area was created.

KEY WORDS: Colombian Caribbean, marine protected areas, fish fauna, artisanal fishing 
La Vía Parque Isla Salamanca, ubicada en la costa norte colombiana (departamento de Magdalena) en jurisdicción de los municipios de Sitionuevo y Puebloviejo, es un área protegida de conservación estricta del Sistema de Parques Nacionales de Colombia (Figura 1). Limita al norte con el mar Caribe, al sur con la Ciénaga Grande de Santa Marta y el Caño Clarín Nuevo, al oriente con el corregimiento de Tasajera (Puebloviejo) y al occidente con el río Magdalena (UAESPNN, 2004). Entre los ecosistemas de la Vía Parque se incluyen las playas de arena; sin embargo, el único estudio de fauna íctica en el área marina se realizó en 2006, cuando la Unidad Administrativa Especial del Sistema de Parques Nacionales adoptó el SIPEIN (Sistema de Información Pesquera del Invemar) como una herramienta para evaluar la presión por pesca sobre los recursos hidrobiológicos y diseñar estrategias de manejo y conservación.

Por otro lado, las áreas marinas y costeras son de gran diversidad, lo que hace que sean considerados uno de los ecosistemas más productivos del planeta (Eichbaum et al., 1996). En Colombia, la biodiversidad íctica es de las más altas de Suramérica (Polanco y Acero, 2020), pero la falta de registros a nivel de especie y la discontinuidad y
Vía Parque Isla Salamanca, located on the Colombian north coast (Magdalena Department), in the jurisdiction of the municipalities of Sitionuevo and Puebloviejo, is a protected area of strict conservation of the Colombian System of National Parks (Figure 1). It is bordered to the north by the Caribbean Sea, to the south by the Ciénaga Grande de Santa Marta and Caño Clarín Nuevo, to the east by the small village of Tasajera (Puebloviejo) and to the west by the Magdalena River (UAESPNN, 2004). The ecosystems of the Vía Parque include sandy beaches; however, the only study of fish fauna in the marine area, was carried out in 2006, when the Special Administrative Unit of the National Parks System adopted the SIPEIN (Invemar's Fisheries Information System) as a tool to assess the pressure of fishing on hydrobiological resources, and to design management and conservation measures.

On the other hand, the marine and coastal areas are of great diversity, making them one of the most productive ecosystems on the planet (Eichbaum et al., 1996). In Colombia, fish biodiversity is one of the largest in South America (Polanco and Acero, 2020), but the lack of records at the species level and the discontinuity and scarce geographical coverage of fisheries data (Chasqui et al., 2017)

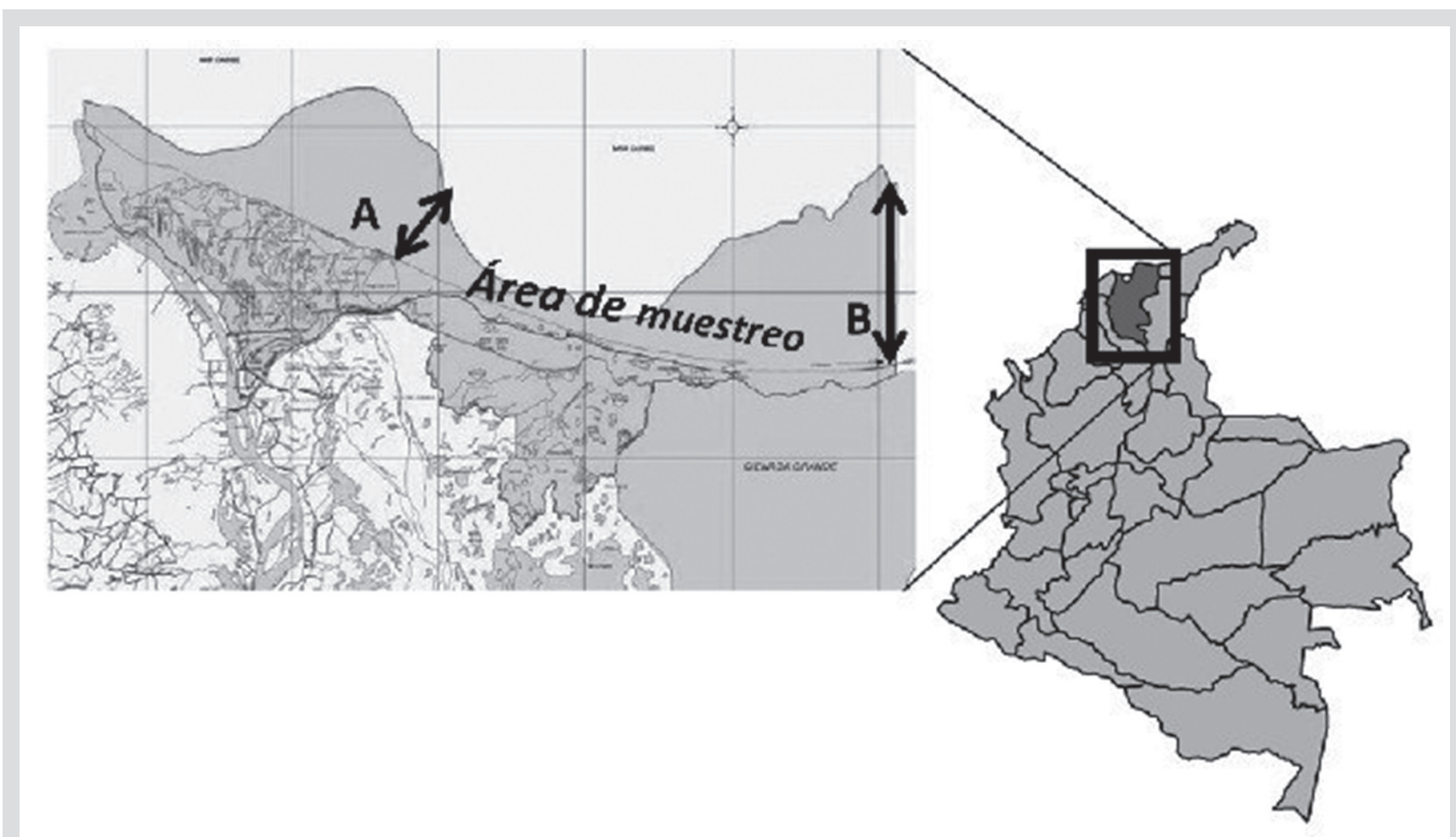

Figura 1. Vía Parque Isla de Salamanca (sector sombreado) y el área de muestreo A-B (línea costera hacia el mar). Fuente: archivo VP Isla Salamanca.
Figure 1. Vía Parque Isla Salamanca (shaded sector) and the sampling area A-B (coastline towards the sea). Source: file VP Isla Salamanca. 
escasa cobertura geográfica de la toma de datos pesqueros (Chasqui et al., 2017), hace que sea necesario avanzar en el estudio y monitoreo de peces marinos, así como en la evaluación de stocks multiespecíficos (Duarte y Schiller, 1997). Esta información permite orientar acciones concretas y propuestas hacia el manejo y la conservación de especies ícticas marino costeras (Manjarrés, 2004).

El poco conocimiento del área marina del Parque y la falta de registros en biodiversidad íctica en el Caribe colombiano, direccionaron este estudio, con miras a conocer el ensamblaje de peces marinos que converge en la VP Isla Salamanca. Para ello, se evaluaron capturas mensuales (entre septiembre de 2006 y abril de 2007), obtenidas con una red de playa conocida como chinchorro (longitud total de la malla: $500 \mathrm{~m}$, ojo de malla: 7,5 cm, longitud total del copo: $8 \mathrm{~m}$, ojo de malla del copo: $5,5 \mathrm{~cm}$ ). Se realizaron 25 lances con pescadores artesanales del corregimiento de Tasajera, quienes tienen la experiencia necesaria en la utilización del aparejo y conocimiento del área de muestreo (Figura 2). Este método es la pesca menos selectiva de la makes it necessary continue studying and monitoring marine fish, as well as assessing multispecies stocks (Duarte and Schiller, 1997). This information allows directing specific actions and management proposals towards the conservation of coastal marine fish species (Manjarrés, 2004).

The little knowledge of the marine area of the Park as well as the lack of records on fish biodiversity in the Colombian Caribbean, lead this study, in order to know the assemblages that occur in the VP Isla Salamanca. Monthly catches were evaluated (between September 2006 and April 2007), obtained with a beach seine known as chinchorro (total mesh length: $500 \mathrm{~m}$, mesh eye $7.5 \mathrm{~cm}$, total length of the cod-end: $8 \mathrm{~m}$, cod-end mesh eye: $5.5 \mathrm{~cm}$ ). Twentyfive (25) hauls were made with artisanal fishermen from the village of Tasajera, who had the necessary experience in using the gear and knowledge of the sampling area (Figure 2). This method is the least selective fishing in the area, and guarantees a greater representation of species and individuals of different sizes.

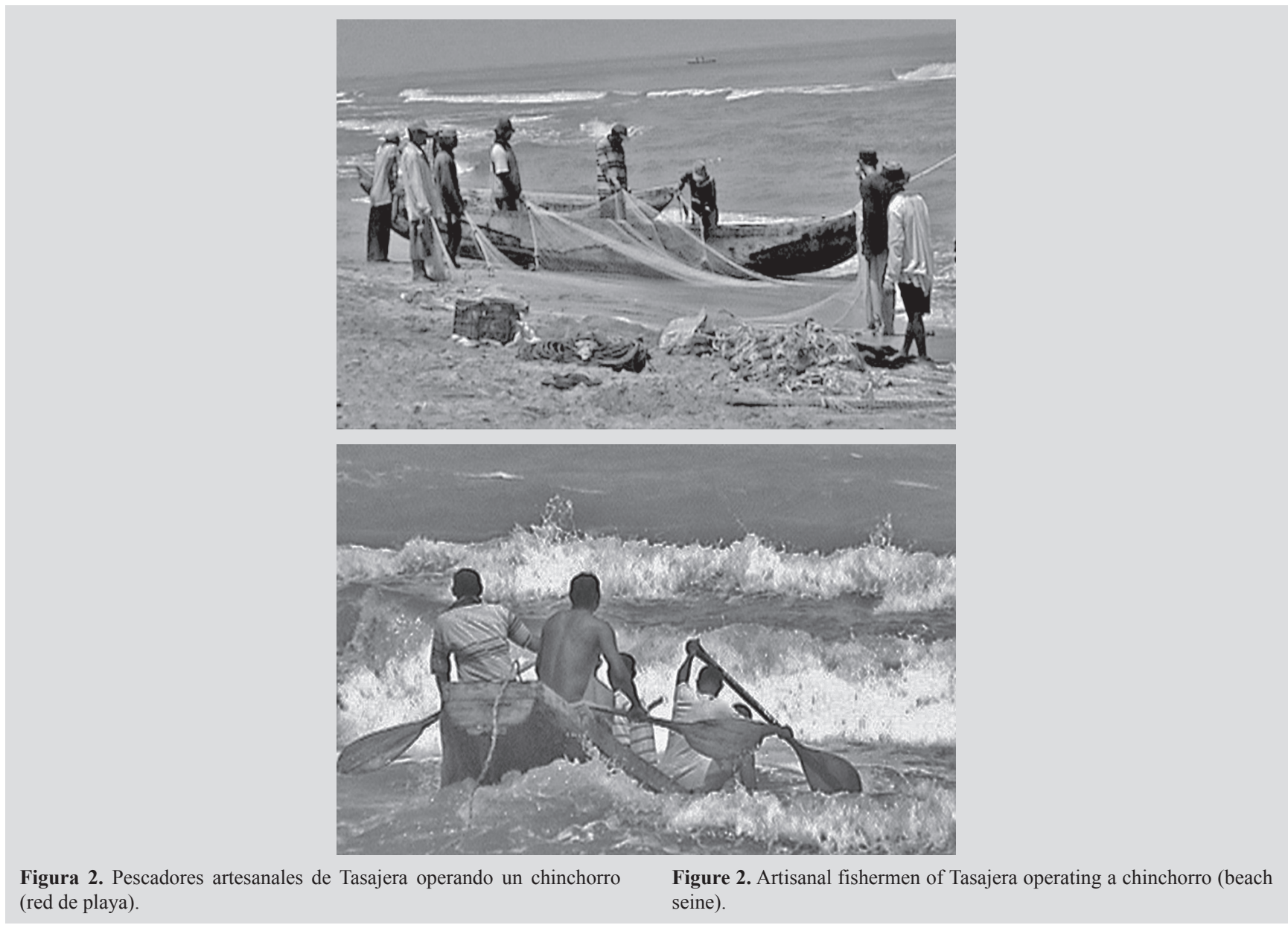


zona, lo que garantiza una mayor representación de especies e individuos de diversas tallas.

Los individuos capturados fueron contados y se identificaron utilizando las guías de Cervigón et al. (1992) y Carpenter (2002) a partir de la observación en el sitio de muestreo, y se revisó la taxonomía y nomenclatura en Robertson et al. (2019) y Fricke et al. (2020). Los peces que no se pudieron identificar en campo fueron recolectados en bolsas plásticas de sello hermético y se transportaron al Invemar para su posterior identificación en el laboratorio.

Se registró un total de $10 \quad 114$ individuos pertenecientes a 16 familias y 31 especies. Las familias Carangidae y Sciaenidae fueron las de mayor representación (Tabla 1). La riqueza encontrada en este estudio es más baja a lo registrado en aguas marinas adyacentes (59 especies; SEPEC, 2019), en aguas estuarinas (87 especies; SantosMartínez y Acero, 1991), y en formaciones del Banco de las Ánimas (85 especies; Acero y García-Urueña, 2020). Estas diferencias pueden explicarse por la frecuencia y duración de los muestreos, así como por la metodología aplicada de acuerdo con los ecosistemas evaluados, demostrando la heterogeneidad ecosistémica en una parte del área marinocostera del departamento de Magdalena. Las mayores coincidencias se obtuvieron con registros realizados en la Ciénaga Grande de Santa Marta (26), evidenciando una diversidad importante, debido a variaciones temporales en abundancia y composición de especies objeto de pesca, ligadas a la variabilidad climática global y asociadas a cambios en la salinidad (Invemar, 2019).
Captured individuals were counted and identified using the guides of Cervigón et al. (1992) and Carpenter (2002) at the sampling site, and the taxonomy was later revised according to Robertson et al. (2019) and Fricke et al. (2020). The fish that could not be identified were collected in hermetic plastic bags and transported to Invemar for identification in the laboratory.

A total of 10114 individuals were collected, belonging to 16 families and 31 species. Families Carangidae and Sciaenidae were the better represented (Table 1). The richness found in this study is lower than that reported in adjacent marine waters (59 species; SEPEC, 2019), from the estuarine area ( 87 species; Santos-Martínez and Acero, 1991), and from the Banco de las Ánimas (85 species; Acero and García-Urueña 2020). Differences may be explained by the frequency and time spam of samplings, as well as by the methodology used in relation to the evaluated ecosystem, demonstrating the ecosystem heterogeneity in a part of coastal-marine area in the north of the Department of Magdalena. The greatest coincidences were with the Ciénaga Grande de Santa Marta (26), evidencing an important diversity, due to temporary variations in abundance and composition of species targeted by fishing, linked to global climate variability, and associated with changes in salinity (Invemar, 2019).

Seven fished species are included in the Colombian Red Book of Marine Fishes (Chasqui et al., 2017); five of them with some category of threat, and only one of them considered Vulnerable globally (IUCN, 2020; Table 2).
Tabla 1. Especies de peces registradas en el área marina de la VP Isla Salamanca y comparación con otros estudios adyacentes al área de estudio: A) SEPEC (2019). B) Zubiría et al. (2009). C) Rueda y Defeo (2003). D) Sánchez y Rueda (1999). E) Santos-Martínez y Acero (1991). F) Invemar (2019). G) Acero y García-Urueña (2020). H) Navas et al. (2017).
Table 1. Fish species registered in the marine area of the VP Isla Salamanca, and comparison with studies adjacent to the study area: A) SEPEC (2019). B) Zubiria et al. (2009). C) Rueda and Defeo (2003). D) Sánchez and Rueda (1999). E) Santos-Martínez and Acero (1991). F) Invemar (2019). G) Acero and García-Urueña (2020). H) Navas et al. (2017)..

\begin{tabular}{|c|c|c|c|c|c|c|c|c|c|}
\hline & \multicolumn{2}{|l|}{ Marino/Marine } & \multicolumn{5}{|c|}{ Estuarino/Estuarine } & \multirow{2}{*}{\multicolumn{2}{|c|}{$\begin{array}{c}\begin{array}{c}\text { Formación } \\
\text { coralina/Coral } \\
\text { formation }\end{array} \\
\begin{array}{c}\text { Banco de las } \\
\text { Ánimas }\end{array}\end{array}$}} \\
\hline & VP Isla Salamanca & $\begin{array}{l}\text { Pueblo } \\
\text { Viejo }\end{array}$ & $\begin{array}{c}\text { VP Isla } \\
\text { Salamanca }\end{array}$ & \multicolumn{4}{|c|}{ Ciénaga Grande de Santa Marta } & & \\
\hline & Este estudio/This study & $\mathbf{A}$ & B & C & D & $\mathbf{E}$ & $\mathbf{F}$ & G & $\mathbf{H}$ \\
\hline $\begin{array}{l}\text { N. }{ }^{\circ} \text { de especies/ } \\
\text { Num. of species }\end{array}$ & 31 & 59 & 37 & 39 & 57 & 87 & 76 & 85 & 69 \\
\hline Familia/Family & Especie/Species & & & & & & & & \\
\hline Rhinopteridae & Rhinoptera brasiliensis (Mitchill, 1815) & $\mathrm{X}$ & & & & & & & \\
\hline Elopidae & $\begin{array}{l}\text { Elops smithi Mcbride, Rocha, Ruíz-Carus y } \\
\text { Bowen, } 2010\end{array}$ & $\mathrm{X}$ & $\mathrm{X}$ & $\mathrm{X}$ & $\mathrm{X}$ & $\mathrm{X}$ & $\mathrm{X}$ & & \\
\hline
\end{tabular}




\begin{tabular}{|c|c|c|c|c|c|c|c|c|c|}
\hline & \multicolumn{2}{|l|}{ Marino/Marine } & \multicolumn{5}{|c|}{ Estuarino/Estuarine } & \multirow{2}{*}{\multicolumn{2}{|c|}{$\begin{array}{c}\begin{array}{c}\text { Formación } \\
\text { coralina/Coral } \\
\text { formation }\end{array} \\
\begin{array}{c}\text { Banco de las } \\
\text { Ánimas }\end{array}\end{array}$}} \\
\hline & VP Isla Salamanca & $\begin{array}{l}\text { Pueblo } \\
\text { Viejo }\end{array}$ & $\begin{array}{l}\text { VP Isla } \\
\text { Salamanca }\end{array}$ & \multicolumn{4}{|c|}{ Ciénaga Grande de Santa Marta } & & \\
\hline & Este estudio/This study & $\mathbf{A}$ & B & $\mathbf{C}$ & D & $\mathbf{E}$ & $\mathbf{F}$ & G & $\mathbf{H}$ \\
\hline Megalopidae & Megalops atlanticus Valenciennes, 1847 & $\mathrm{X}$ & $\mathrm{X}$ & $\mathrm{X}$ & $\mathrm{X}$ & $\mathrm{X}$ & $\mathrm{X}$ & & \\
\hline Clupeidae & Opisthonema oglinum (Lesueur, 1818) & $\mathrm{X}$ & & $\mathrm{X}$ & $\mathrm{X}$ & $\mathrm{X}$ & $\mathrm{X}$ & & \\
\hline Engraulidae & Anchovia clupeoides (Swainson, 1839) & & $\mathrm{X}$ & $\mathrm{X}$ & $\mathrm{X}$ & $\mathrm{X}$ & $\mathrm{X}$ & & \\
\hline \multirow{2}{*}{ Ariidae } & Bagre marinus (Mitchill, 1815) & & & & & $\mathrm{X}$ & $\mathrm{X}$ & & \\
\hline & Sciades proops (Valenciennes, 1840) & $\mathrm{X}$ & & & & $\mathrm{X}$ & $\mathrm{X}$ & & \\
\hline \multirow{2}{*}{ Scombridae } & $\begin{array}{l}\text { Scomberomorus brasiliensis Collette, Russo y } \\
\text { Zavala-Camin, } 1978\end{array}$ & $\mathrm{X}$ & & $\mathrm{X}$ & & $\mathrm{X}$ & & & \\
\hline & Scomberomorus cavalla (Cuvier, 1829) & $\mathrm{X}$ & & & & & & & \\
\hline Centropomidae & Centropomus undecimalis (Bloch, 1792) & $\mathrm{X}$ & $\mathrm{X}$ & $\mathrm{X}$ & $\mathrm{X}$ & $\mathrm{X}$ & $\mathrm{X}$ & & \\
\hline \multirow{2}{*}{ Sphyraenidae } & Sphyraena barracuda (Edwards, 1771) & & & & & & $\mathrm{X}$ & $\mathrm{X}$ & \\
\hline & Sphyraena guachancho Cuvier, 1829 & $\mathrm{X}$ & & & & & & & \\
\hline Achiridae & Achirus lineatus (Linnaeus, 1758) & & $\mathrm{X}$ & $\mathrm{X}$ & $\mathrm{X}$ & $\mathrm{X}$ & $\mathrm{X}$ & & \\
\hline \multirow{6}{*}{ Carangidae } & Caranx crysos (Mitchill, 1815) & $\mathrm{X}$ & & & & & $\mathrm{X}$ & & \\
\hline & Caranx hippos (Linnaeus, 1766) & $\mathrm{X}$ & $\mathrm{X}$ & $\mathrm{X}$ & $\mathrm{X}$ & $\mathrm{X}$ & $\mathrm{X}$ & & \\
\hline & Oligoplites saurus (Bloch y Schneider, 1801) & & $\mathrm{X}$ & $\mathrm{X}$ & $\mathrm{X}$ & $\mathrm{X}$ & $\mathrm{X}$ & & \\
\hline & Selene setapinnis (Mitchill, 1815) & & & & & & $\mathrm{X}$ & & \\
\hline & Selene vomer (Linnaeus, 1758) & & & & & $\mathrm{X}$ & $\mathrm{X}$ & & \\
\hline & Trachinotus carolinus (Linnaeus, 1766) & $\mathrm{X}$ & & & & $\mathrm{X}$ & $\mathrm{X}$ & & \\
\hline \multirow{2}{*}{ Mugilidae } & Mugil incilis Hancock, 1830 & $\mathrm{X}$ & $\mathrm{X}$ & $\mathrm{X}$ & $\mathrm{X}$ & $\mathrm{X}$ & $\mathrm{X}$ & & \\
\hline & Mugil liza Valenciennes, 1836 & $\mathrm{X}$ & $\mathrm{X}$ & $\mathrm{X}$ & $\mathrm{X}$ & $\mathrm{X}$ & $\mathrm{X}$ & & \\
\hline Ephippidae & Chaetodipterus faber (Broussonet, 1782) & & $\mathrm{X}$ & $\mathrm{X}$ & $\mathrm{X}$ & $\mathrm{X}$ & $\mathrm{X}$ & & \\
\hline Gerreidae & Diapterus rhombeus (Cuvier, 1829) & $\mathrm{X}$ & $\mathrm{X}$ & $\mathrm{X}$ & $\mathrm{X}$ & $\mathrm{X}$ & $\mathrm{X}$ & & \\
\hline \multirow{2}{*}{ Haemulidae } & Conodon nobilis (Linnaeus, 1758) & $\mathrm{X}$ & & & & $\mathrm{X}$ & $\mathrm{X}$ & & \\
\hline & Haemulon bonariense Cuvier, 1830 & $\mathrm{X}$ & & & & & & & \\
\hline \multirow{6}{*}{ Sciaenidae } & Larimus breviceps Cuvier, 1830 & $\mathrm{X}$ & & $\mathrm{X}$ & & $\mathrm{X}$ & $\mathrm{X}$ & & \\
\hline & Menticirrhus littoralis (Holbrook, 1847) & $\mathrm{X}$ & & & & $\mathrm{X}$ & $\mathrm{X}$ & & \\
\hline & Micropogonias furnieri (Desmarest, 1823) & $\mathrm{X}$ & & $\mathrm{X}$ & $\mathrm{X}$ & $\mathrm{X}$ & $\mathrm{X}$ & & \\
\hline & Paralonchurus brasiliensis (Steindachner, 1875) & & & & & $\mathrm{X}$ & $\mathrm{X}$ & & \\
\hline & Stellifer venezuelae (Schultz, 1945) & & $\mathrm{X}$ & $\mathrm{X}$ & $\mathrm{X}$ & $\mathrm{X}$ & $\mathrm{X}$ & & \\
\hline & Umbrina coroides Cuvier, 1830 & $\mathrm{X}$ & & & & $\mathrm{X}$ & $\mathrm{X}$ & & \\
\hline \multicolumn{2}{|c|}{ Total de coincidencias/Total matches } & 21 & 12 & 16 & 14 & 24 & 26 & 1 & 0 \\
\hline
\end{tabular}


Siete especies están incluidas en el Libro rojo de peces marinos de Colombia (Chasqui et al., 2017), cinco de ellas con alguna categoría de amenaza, solo una de ellas considerada como Vulnerable en el ámbito global (IUCN, 2020; Tabla 2). Los criterios de identificación de amenazas varían según la escala regional, nacional y global que se esté manejando, teniendo en cuenta los factores antropogénicos a los cuales estén siendo sometidas las poblaciones. Para el caso de las especies marinas, presentan diferentes presiones como la sobreexplotación de los recursos, uso de artes de pesca inadecuadas, desarrollo desordenado de actividades en zonas costeras, contaminación por vertimientos de aguas servidas al mar, aumento en el tráfico marítimo, actividad turística desmedida e insostenible, falta de legislación clara en temas marino costeros, entre otros (Chasqui et al., 2017).

Tabla 2. Especies registradas en el área marina de la VP Isla Salamanca e incluidas en el Libro Rojo nacional (Chasqui et al., 2017) y en la lista roja global (IUCN, 2020).
Threat identification criteria vary in regional, national and global scales, taking into account anthropogenic factors to which fish populations are being exposed. In the case of marine species, different pressures have been mentioned, such as resource overexploitation, use of inappropriate fishing gear, chaotic developments in coastal areas, direct sewage discharge into the sea, increase in maritime traffic, excessive and unsustainable tourist activity, and lack of clear legislation on coastal marine issues, among others (Chasqui et al., 2017).

These results contribute to take management proposals towards the protection of hydrobiological resources in the marine area of the Vía Parque. Some of them are articulate conservation measures for mangrove areas adjacent to the marine area of the Park, proposing

Table 2. Species registered in the marine area of the VP Isla Salamanca and included in the National Red Book (Chasqui et al., 2017) and the global Red List (IUCN, 2020).

\begin{tabular}{|l|c|c|}
\hline \multicolumn{1}{|c|}{ Especie/Species } & $\begin{array}{c}\text { Categoría nacional/Nacional status } \\
\text { Chasqui } \text { et al. (2017) }\end{array}$ & $\begin{array}{c}\text { Categoría global/Global status } \\
\text { IUCN (2020) }\end{array}$ \\
\hline Megalops atlanticus & CR En Peligro Crítico/Critically endangered & VU Vulnerable \\
Caranx hippos & VU Vulnerable & LC Preocupación Menor/Least concern \\
\hline Centropomus undecimalis & VU Vulnerable & LC Preocupación Menor/Least concern \\
\hline Mugil incilis & VU Vulnerable & LC Preocupación Menor/Least concern \\
\hline Sciades proops & VU Vulnerable & NE No Evaluado/Not evaluated \\
Sphyraena barracuda & NT Casi Amenazada/Near threatened & LC Preocupación Menor/Least concern \\
\hline Mugil liza & NT Casi Amenazada/Near threatened & DD Datos Insuficientes/Data deficient \\
\hline
\end{tabular}

Con estos resultados es posible realizar propuestas de manejo hacia la protección de los recursos hidrobiológicos en el área marina de la Vía Parque; entre ellas articular medidas de conservación de las áreas de manglar adyacentes al área marina del Parque, proponer estudios de distribución, biología y ecología de las especies ícticas, establecimiento de vedas y de las tallas mínimas de captura para las especies ícticas no comerciales, y gestión interinstitucional para realizar educación ambiental, para cumplir con la misión y gestión de conservación para la cual ha sido creada esta área protegida. studies of distribution, biology and ecology of fish species, establishing closures and minimum catch sizes for noncommercial fish species, and developing interinstitutional management to carry out environmental education; the mission and conservation management for which this protected area has been created will be then fulfilled.

\section{ACKNOWLEDGMENTS}

This work was part of the first author thesis to obtain the degree of Biologist at the University of Tolima, and had the support of the Administrative Unit of National Parks. The authors thank Professors Jacobo Blanco (RIP), 


\section{AGRADECIMIENTOS}

Este trabajo hizo parte del proyecto de grado de la primera autora para obtener el título de Bióloga en la Universidad del Tolima, y contó con el apoyo de la Unidad Administrativa de Parques Nacionales Naturales de Colombia. Los autores agradecen a los profesores Jacobo Blanco (q. e. p. d.), Juan Carlos Narváez y Héctor Fabio Sáenz por sus aportes; a los pescadores de Tasajera, Magdalena, por su colaboración en el uso del chinchorro, y a los evaluadores anónimos por sus correcciones y sugerencias. Contribución 507 del Cecimar y 1287 del Invemar.
Juan Carlos Narváez, and Héctor Fabio Sáenz for their help, the fishermen of Tasajera-Magdalena for their collaboration in the using the chinchorro, and the anonymous reviewers for their corrections and suggestions. Contribution 507 from Cecimar and 1287 from Invemar.

\section{BIBLIOGRAFÍA/LITERATURE CITED}

Acero P., A. y R. García-Urueña. 2020. Peces del Banco de las Ánimas. 25-40. En: García-Urueña, R. (Ed.). El Banco de las Ánimas: valoración de su biodiversidad. Univ. Magdalena, Santa Marta. 136 p.

Carpenter, K.E. (Ed.). 2002. Species identification guide for fishery purposes the living marine resources of the western central Atlantic. FAO, Rome. 2127 p.

Cervigón, F., R. Cipriani, W. Fischer, L. Garibaldi, M. Hendrickx, A.J. Lemus, R. Márquez, J.M. Poutiers, G. Robaina y B. Rodríguez. 1992. Fichas FAO de identificación de especies para los fines de pesca. Guía de campo de las especies comerciales marinas y de aguas salobres de la costa septentrional de Sur América. FAO, Roma. 513 p.

Chasqui V., L., A. Polanco F., A. Acero P., P.A. Mejía-Falla, A. Navia, L.A. Zapata, y J.P. Caldas. (Eds.). 2017. Libro rojo de peces marinos de Colombia. Ser. Publ. Gen. Invemar, (93), $552 \mathrm{p}$.

Duarte, Ly D. von Schiller. 1997. Comunidad de peces demersales del Golfo de Salamanca (Caribe colombiano): estructura espacio-temporal y caracterización trófica con énfasis en los hábitos alimenticios de Lutjanus analis (Cuvier, 1828), Lutjanus synagris (Linnaeus, 1758), Balistes capriscus (Gmelin, 1788) y Balistes vetula (Linneaus, 1758). Tesis Biol. Mar., Univ. de Bogotá Jorge Tadeo Lozano, Santa Marta. 184 p.

Eichbaum, W.M., M.P. Crosby, M.T. Agardy, and S.A. Laskin. 1996. The role of marine and coastal protected areas in the conservation and sustainable use of biological diversity. Ocenography, 9(1): 60-70.

Fricke, R., W.N. Eschmeyer, and R. Van der Laan (Eds.). 2020. Eschmeyer's catalog of fishes: genera, species, references. http://researcharchive.calacademy. org/research/ichthyology/catalog/fishcatmain.asp. 12/09/2020.

Invemar. 2019. Monitoreo de las condiciones ambientales y los cambios estructurales y funcionales de las comunidades vegetales y de los recursos pesqueros durante la rehabilitación de la Ciénaga Grande de Santa Marta. Informe técnico final. Invemar, Santa Marta. 214 p.

IUCN. 2020. The IUCN red list of threatened species. Version 2020-2. https://www.iucnredlist.org. 17/10/2020.

Manjarrés, L. 2004. Estadísticas pesqueras artesanales de los departamentos de Magdalena y La Guajira, con aplicación de herramientas informáticas para su sistematización y procesamiento. Universidad Magdalena-INPA-Colciencias, Santa Marta. 72 p.

Navas, R., A. Acosta, L. Sánchez, J. González, M. Ontiveros, J.A. Rodríguez-Rodríguez y P. Obando. 2017. Caracterización del ecosistema asociado al sector de las Ánimas, departamento del Magdalena. Anexo 4. Convenio de cooperación n. ${ }^{\circ}$ 005-2016-PNSA-Invemar, Santa Marta.

Polanco F., A. y A. Acero P. 2020. Biodiversidad de peces marinos del Caribe colombiano. 23-27. En: Conservación Internacional Colombia y WWF. La pesca en Colombia: del agua a la mesa. Conservación Internacional Colombia y WWF, Envigado. 98 p.

Robertson, D.R., E.A. Peña, J.M. Posada y R. Claro. 2019. Peces costeros del Gran Caribe: sistema de información en línea. Version 2.0. Inst. Smithsonian Invest. Trop., Balboa, Panamá. https://biogeodb.stri.si.edu/caribbean

Rueda, M. and O. Defeo. 2003. Spatial structure of fish assemblages in a tropical estuarine lagoon: combining multivariate and geostatistical techniques. J. Exp. Mar. Biol. Ecol., 93-112.

Sánchez, C. y Rueda, M. 1999. Variación de la diversidad y abundancia de especies ícticas dominantes en el delta del río Magdalena, Colombia. Rev. Biol. Trop., 47(4): 1067-1079. 
Santos-Martínez, A. and A. Acero P. 1991. Fish community of the Ciénaga Grande de Santa Marta (Colombia) composición and zoogeography. Ichthyol. Explor. Freshwaters, 2(3): 247-263.

SEPEC. 2019. Servicio estadístico pesquero colombiano. http://sepec.aunap.gov.co/. 13/09/2020.

UAESPNN. 2004. Plan de manejo Vía Parque Isla de Salamanca 2005-2009. Parques Nacionales Naturales de Colombia, Barranquilla. 136 p.

Zubiria, W., A. Lacayo, A. Acero P. y J. Narváez. 2009. Diversidad y abundancia de la ictiofauna de un complejo de lagunas costeras en una reserva natural del Caribe colombiano. Mem. Fund. La Salle Cienc. Nat., 170: 125-139.

RECIBIDO/RECEIVED: 01/08/2020

ACEPTADO/ACCEPTED: 08/11/2020 